Review

\title{
[INVITED] New advances in fiber cavity ring-down technology is
}

\author{
S.O. Silva, R. Magalhães, M.B. Marques, O. Frazão* \\ INESC TEC and Department of Physics and Astronomy, Faculty of Sciences of University of Porto, Rua do Campo Alegre 687, 4169-007 Porto, Portugal
}

\section{A R T I C L E I N F O}

\section{Article history:}

Received 28 August 2015

Accepted 12 October 2015

\section{Keywords:}

Cavity-ring down

Optical fiber sensor

Applications

\begin{abstract}
A B S T R A C T
A brief review in the cavity ring-down technique (CRD) is presented. In this review, there will only be considered the conventional fiber CRD configuration, i.e., there will only be presented researches involving cavities with two couplers with 99:1 ratios, due to the large amount of publications involving this spectroscopy method. The presented survey is divided in different topics related to the measurement of physical parameters, such as strain and temperature, curvature, pressure, refractive index, gas and biochemical sensing.
\end{abstract}

(c) 2015 Elsevier Ltd. All rights reserved.

\section{Contents}

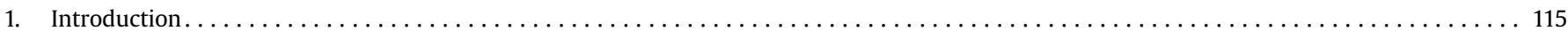

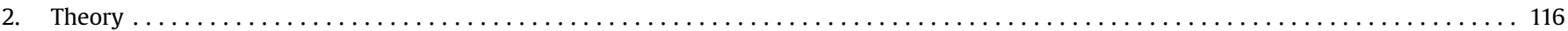

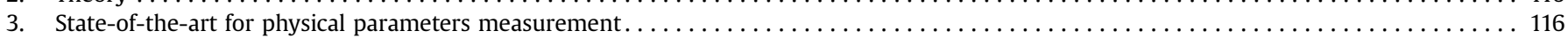

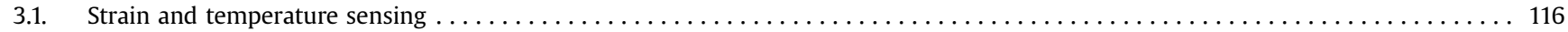

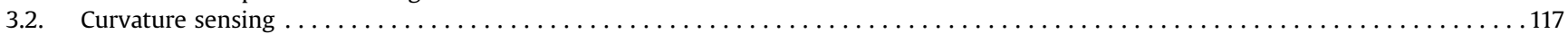

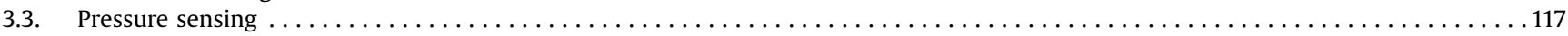

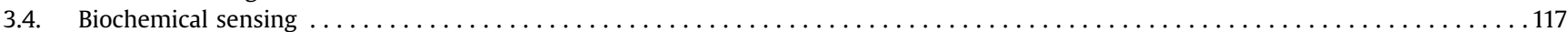

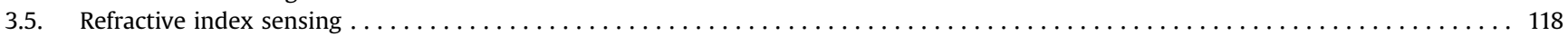

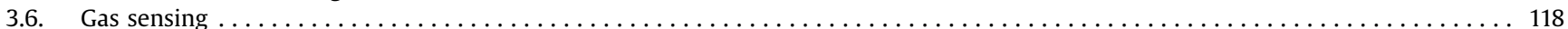

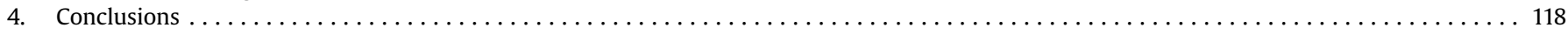

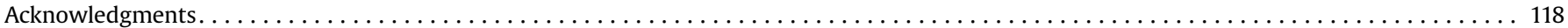

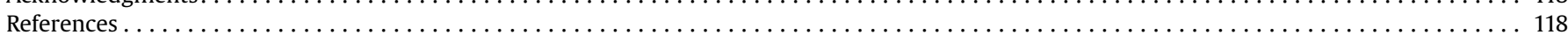

\section{Introduction}

The cavity ring-down (CRD) technique consists in a spectroscopy method broadly used in technology. Being mainly employed in the sensor area, this methodology enables an accurate analysis of amplitude behavior over the time. Throughout the past decades, the CRD spectroscopy has been subject of a lot of research, namely in chemical and molecular analysis in real time [1]. The principle involved settles the basis for various configurations, being also applied to resonant optical cavities with high reflective mirrors, as a result of the high evolution of this technique $[2,3]$. Through

\footnotetext{
${ }^{2}$ Invited Article by the Associate editor: Christophe Caucheteur.

* Corresponding author. Fax: +351 220402437.

E-mail addresses: sfsilva@inescporto.pt (S.O. Silva),

regina.t.magalhaes@inesctec.pt (R. Magalhães),

mbmarque@fc.up.pt (M.B. Marques), ofrazao@inescporto.pt (O. Frazão).
}

association with the previous developed fiber loops, there were implemented new fiber optic-based CRD settings, which, in turn, used a fiber loop operating as the resonant cavity. This configuration quickly obtained a lot of popularity in the scientific community, mainly for presenting an effective alternative to the usual CRD configuration [4]. By virtue of these conceptual studies, the CRD technique has been target of a lot of research in the spectroscopy field, being implemented in the last decade to the measurement of physical parameters, such as strain [5-7], temperature [8], curvature $[9,10]$ and pressure [11-13]. More recently, a fast development of CRD technique allied to the biochemical sensing field can also be easily recognized. The implementations in this area are quite extensive, which can go from detection of organic dyes [14] to unicellular organisms [15], or even 1-octyne in decane solution [16]. The CRD approach has also been widely used for refractive index of liquids [17-21] and gas sensing [22-25].

Along with the expansion in these areas, the CRD technique has 
also been practically reinvented, yielding the investigation of other areas, such as cavity-enhanced spectroscopy [4]. Due to the broad investigation, this technique has been extended being so reported many different functions using a Fiber Loop Ringdown (FLRD) [26]. Opening a micro hole in one of the fiber end faces, a significant reduction in the coupling loss was achieved [27]. In addition to these applications, the CRD technique use is widespread, being implemented in chemical sensors [28], using frequency-shifted interferometry [29], using wavelength-tunable ultrashort pulsed light [30] or even an Optical Time Domain Reflector (OTDR) instead of the usual implemented laser and modulator setup [31]. More recently, a new CRD topology for remote Sensing was demonstrated [32].

The employability of this technique is very wide, offering great flexibility and conferring immense possibilities on its use, such as in fiber loop sensing devices (including fiber Bragg gratings, long period gratings, fiber microchannels and photonic crystal fibers). These elements, when put together with the CRD technique, have allowed high resolution and high sensitive values, yielding huge developments in the optical fiber industry. Thus, pulses of high intensity and highly sensitive photodetectors or optical amplifiers within the cavity are commonly used. The spectroscopy method has shown great potential for high sensitivity detection, which led to a very wide range of applications in research laboratories. For this reason, a revision of the work made implementing these conventional structures is presented, in order to elaborate a complete study on this subject.

\section{Theory}

A fiber cavity ring-down operation lays down on a simple principle. The measurements obtained by adopting a conventional cavity ring-down technique are related to the injection of pulses into an extremely high-finesse bulk cavity, which can be achieved either through the use of reflective layers $(R>99.9 \%)$ or simply by using fiber couplers of high split ratios (e.g. 99:1) [27]. In this review, there will only be considered the conventional fiber CRD configuration, i.e., there will only be studied cavities with two couplers with a 99:1 ratio (see Fig. 1). That way, the revision made is limited to the existent research involving this type of conventional structures. As represented in Fig. 1, the configuration operates as follows: port 1 is coupled in with port 3 , by means of the first coupler, being the output port 4 recoupled as an input signal (port 5) with the input port 7, through the second coupler. Port 6 and port 3 are connected, forming the cavity ring-down structure. Port 8 is used as an output arm to interrogate the amplitude of the signal over time. The two couplers operate in distinct ways; one for the source and the other for measuring the intensity of the

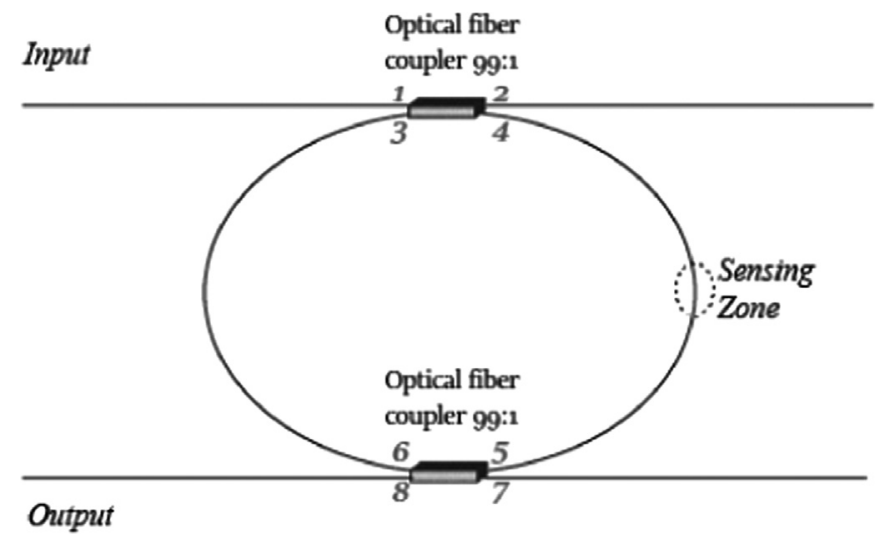

Fig. 1. Conventional cavity ring-down configuration. pulse, thus forming the analog of the mirrors in a traditional CRD. In the case of time resolved ring-down signals, the initially received intensity is very low, becoming even less with each round trip, due to the high split ratio of the couplers. However, the high reflectivity or coupling ratios are necessary to achieve large numbers of round trips (traveling more time inside the cavity).

The most important aspect in CRD technique is the independence of the output signal in relation to the power of the input signal, so that there is only output signal dependence with time. Due to the losses in the couplers and the fiber intrinsic attenuation, the pulse will slowly decay as it travels around the loop. The rate of decay of the output pulse train indicates the cavity loss. If the losses are not too significant, although only $1 \%$ of the input signal enters the cavity on the first trip, the pulse will still take several turns around the loop, being measured at a given position. Analyzing the signal through port 8, one then observes a series of pulses equally spaced in time, although with increasingly smaller intensities. This time frame can be directly obtained by the formula $t=n L / c$, where $L$ is the length of the cavity, $n$ the effective refractive index of the fiber and $c$ the light velocity.

Let us consider the case when an intensity sensor, $T_{s}$, is placed inside the fiber loop, for the purpose of measuring a certain physical quantity. At every turn traveled by the pulse, it suffers attenuation by the same factor, caused by the fiber intrinsic attenuation, the couplers insertion losses $T_{c}$, and the sensor transmission $T_{s}$. At the end of the first loop, the relation between the intensity of the pulse after the first turn, $I_{1}$, and the initial one, $I_{0}$, is given by [14]:

$\frac{I_{1}}{I_{0}}=T_{S} T_{c}^{2} e^{-\alpha L}$

After $n$ turns, considering $t_{n}$ the time for $n$ turns, the relation between the intensity of the pulse, $I_{n}$, and the initial one is:

$\frac{I_{n}}{I_{0}}=\left(\frac{I_{1}}{I_{0}}\right)^{n}=e^{\left(t_{n} / \Delta t\right) \ln \left(\frac{I_{1}}{I_{0}}\right)}=e^{-t_{n} / \tau}$

The time constant, $\tau$, strongly depends on the losses in the fiber loop [14]:

$\tau=\frac{L}{c\left[\alpha L-\ln T_{S} T_{c}^{2}\right]}$

The principle involved in these sensing measurements are based on the dependence of the decay time with the sensor transmission. Since the pulse travels several times through the intensity sensor, high sensitivities can be achieved by using this technique. Fig. 2 shows the conceptual behavior of the amplitude of the pulse over time, expected on a conventional CRD configuration.

\section{State-of-the-art for physical parameters measurement}

\subsection{Strain and temperature sensing}

In 2004, Tarsa et al. [5] presented a CRD technique with a biconical tapered fiber incorporated in the fiber loop for strain sensing. It was demonstrated a minimum detectable change in ringdown time of $0.08 \%$, corresponding to a minimum detectable displacement of $4.8 \mathrm{~nm}$, and a sensitivity to strain as small as $79 \mathrm{n} \varepsilon / \sqrt{ } \mathrm{Hz}$ over a $5-\mathrm{mm}$ taper length. Later, Ni et al. [6] used a long period grating instead inside the fiber loop to measure strain. By detecting the ringdown time at different strain levels, a high sensitivity of $1.261 \mathrm{~ns} / \mu \varepsilon$ and a minimum detectable strain level of $9 \mu \varepsilon$ could be achieved. Most recently, our research group [7] developed a CRD configuration for the measurement of strain. An 


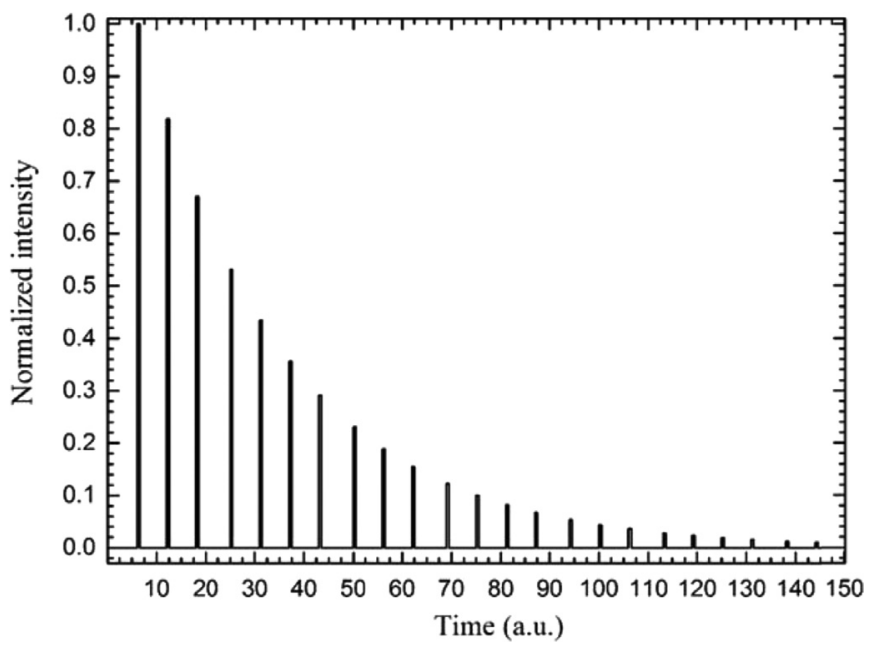

Fig. 2. Normalized cavity ring-down trace, expected for conventional configuration.

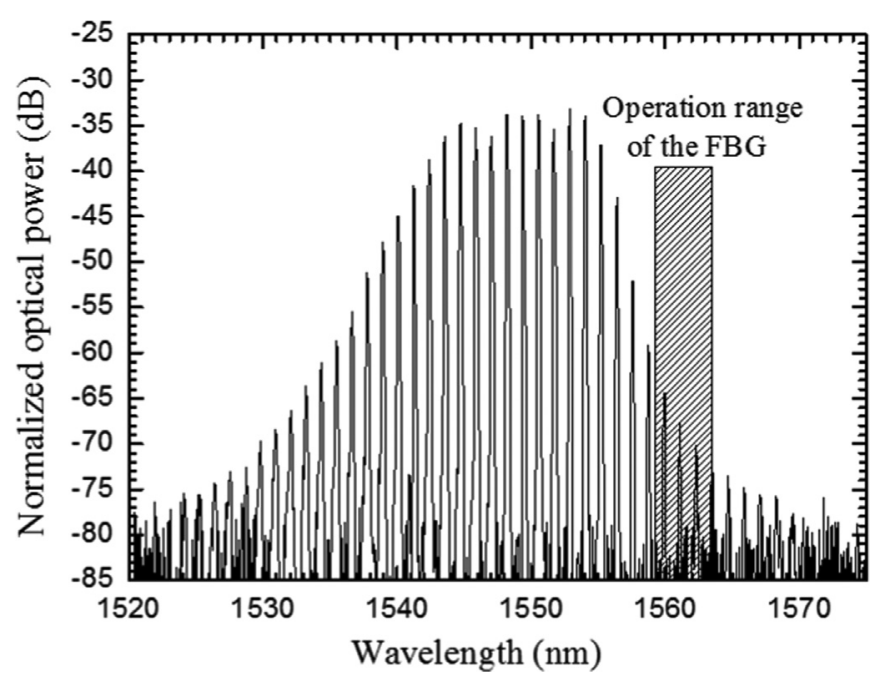

Fig. 3. Optical spectrum of the multimode laser source of the OTDR used in the proposed CRD chirped-FBG [7].

optical time-domain reflectometer (OTDR) was used to send impulses down into the fiber loop cavity, inside of which a chirped fiber Bragg grating (CFBG) was placed to act as a strain sensing element (see Fig. 3). This technique could provide strain results with both conventional CRD-based configuration and the OTDR.

The CRD configuration provided a linear response to strain applied in the range $0-2000 \mu \varepsilon$, and a sensitivity of $1.34 \mathrm{~ns} / \mu \varepsilon$ was obtained. For the same operation range, the OTDR interrogation allowed obtaining a periodic behavior due to CFBG scanning of the wavelength peaks of the multimode laser source while increasing strain. It was shown that the OTDR interrogation method provided a ringdown time response of about $8 \mu$ s which was a great improvement when compared to the $23 \mu$ s-ringdown time obtained with the conventional CRD configuration.

The only attempt to measure temperature was in 2005, when Wang et al. [8] placed a fiber Bragg grating (FBG) inside the fiber loop of a CRD-based configuration - temperature sensing up to $593{ }^{\circ} \mathrm{C}$ was demonstrated using a proof-of-concept device. The ringdown time at room temperature $\left(24^{\circ} \mathrm{C}\right)$ was $4.4 \mu \mathrm{s}$ and dropped to $3.5 \mu \mathrm{s}$ when the FBG amplitude changed with increasing temperature until $68^{\circ} \mathrm{C}$. When the FBG was heated to $593^{\circ} \mathrm{C}$, a ringdown time change of $0.8 \mu$ s was also observed.

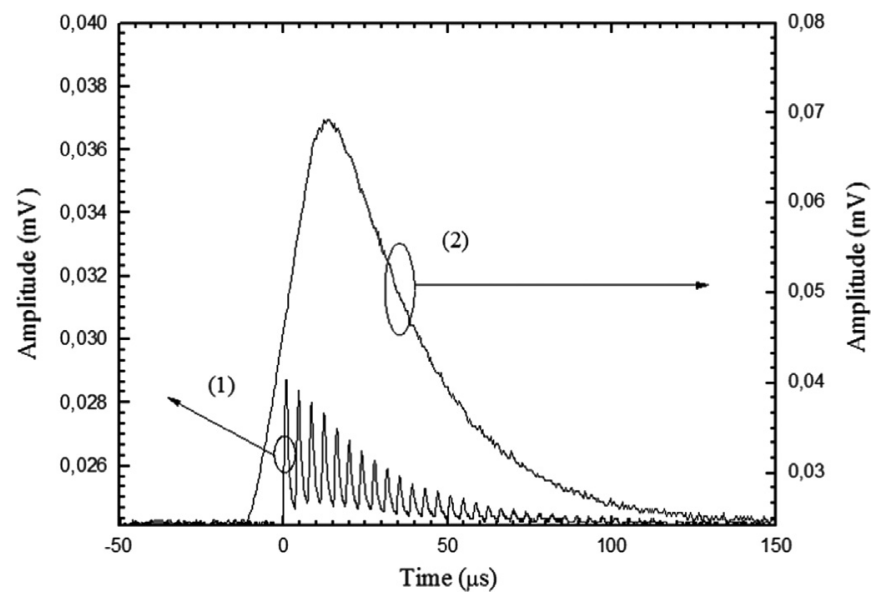

Fig. 4. Cavity ring down trace for (1) conventional configuration using an LPG as sensing head and (2) added-signal obtained by the sum of several conventional CRD impulses.

\subsection{Curvature sensing}

In 2002, Lerber et al. [9] presented a CRD configuration for curvature sensing as a proof-of-concept. A $1.11 \mathrm{~m}$-long singlemode fiber with high-reflection coatings at both ends was chosen for investigating bending-induced losses. A sensitivity of $108 \mathrm{ppm}$ $\left(4.68 \times 10^{-4} \mathrm{~dB}\right)$ of transmission change was achieved. Our group presented recently a CRD scheme using an added-signal for curvature sensing [10]. In this case, a long period grating (LPG) was used as sensing device; an OTDR was used to send impulses down into the fiber loop. The added-signal was obtained by the sum of several conventional CRD impulses, thus providing an improvement on the curvature sensitivity when compared to the conventional CRD signal processing (see Fig. 4). A linear response was observed in the curvature range [2.2-3.6] $\mathrm{m}^{-1}$ and a sensitivity of $15.3 \mu \mathrm{s} / \mathrm{m}^{-1}$ was obtained. This result was found to be 20 -fold the one obtained for the conventional CRD signal processing. The added-signal increased the optical power but increased as well the ringdown time due to the sum of the several loops that light traveled inside the fiber ring. A ringdown time response of $43.3 \mu$ s was attained (vs. $23.7 \mu$ s for the conventional CRD signal processing).

\subsection{Pressure sensing}

In 2004, Wang et al. [11,12] reported an exploratory study on a novel fiber ringdown pressure sensor, showing good performances in terms of stability, repeatability, and dynamic range. The dynamic range for pressure measurement was $6.8 \times 10^{6}-18 \times 10^{6} \mathrm{~Pa}$ as the fiber jacket remained in the sensor head. A few years later, Qiu et al. [13] proposed a CRD configuration based on multimode fiber instead for pressure measurement. The multimode fiber was placed inside the fiber loop to act as pressure sensor, presenting higher sensitivity and larger dynamic range - up to $6.2 \times 10^{7} \mathrm{~Pa}-$ compared to the singlemode counterpart $[11,12]$. Also good stability and repeatability of the sensor was observed.

\subsection{Biochemical sensing}

In 2002, Brown et al. [14] made the first attempt to develop a fiber loop ringdown method which resembled to the traditional bulk cavity ringdown absorption spectroscopy technique. A pulsed, visible and near-infrared laser light was coupled into an optical fiber, which was wound into a loop using a fiber splice connector. The concept was demonstrated through the measurement of both transmission efficiency of an optical splice and absorption of light 
by a liquid sample inserted into the fiber optical loop. The setup successfully measured the solution of an organic dye that was placed between the fiber ends; an absorption spectrum of $7 \times 10^{-15} \mathrm{~mol}$ of the dye $1,1^{\prime}$-diethyl-4,4'-dicarbocyanine iodide in $7 \times 10^{-12}$ L of dimethylsulfoxide was obtained. In 2004, Tarsa et al. [15] implemented a CRD configuration using two optical couplers and a fiber loop cavity for label-free detection of biological species. A chemically etched tapered fiber place inside the fiber ring was used as sensing element which enabled single-cell sensitivity arising from optical scattering of the evanescent field surrounding the tapered fiber. The observed detection limit, based on the minimum detectable loss measured in the system, shown a detectable total scattering cross section of $10 \mu \mathrm{m}^{2}$ for a particle the same size as the mammalian sample cells. For the detection of a single mammalian cancer cell, the measured change in ringdown time was of $0.23 \mu \mathrm{s} /$ cell. Later, Pu et al. [16] demonstrated the use of an LPG inside the CRD fiber ring as chemical sensor by means of evanescent wave interaction with the surrounding medium. The LPG was submerged into a solution of 1-octyne in decane and measured the ringdown pulse trains. It was observed that the decay time decreased with the increase of the analyte concentration.

\subsection{Refractive index sensing}

In 2008, Ni et al. [17] used a Long Period Grating (LPG) that was placed inside the fiber ring and studied the ring-down decay time of the fiber loop as a function of the refractive index (RI) variation of the external medium. Results indicated an amplitude signal decay of $-7.16 \mathrm{~dB}$ in the RI range of $1.35-1.43$, corresponding to a ring-down time of $27.03 \mu$ s and a net loss of the CRD loop of $-0.69 \mathrm{~dB}$. Zhou et al. [18] used in turn a tilted fiber Bragg grating as sensing element inside the fiber loop; using this configuration, a maximum sensitivity of $154 \mu \mathrm{s} / \mathrm{RIU}$ in the high RI range was obtained and a resolution of $6.5 \times 10^{-6}$ RIU. The same research group produced also a micrometric channel that was inscribed in the fiber of the cavity ring by means of femtosecond technique [19]. This microchannel was used to measure gels with different RIs. This configuration allowed obtaining a ring down time with a nonlinear increase as a function of the RI of the gel; a sensitivity up to $300 \mu \mathrm{s} / \mathrm{RIU}$ close to the RI of 1.452 was obtained and a resolution of $1.4 \times 10^{-4}$ RIU. In 2010 , Wang et al. [20] used a partially etched cladding singlemode fiber as sensing element inside the fiber ring. A maximum detection limit for an RI change of $3.2 \times 10^{-5}$ was demonstrated in the RI range of $1.3388-1.3398$; in this RI range, ringdown times between 8.8 and $9.6 \mu$ s were also obtained. Recently, Wong et al. [21] integrated in the cavity ring a Mach-Zehnder interferometer based on Photonic Crystal Fiber (PCF), for the detection of different RIs of liquids. This configuration presented a linear sensitivity of $11.7 \mu \mathrm{s}^{-1} \mathrm{RIU}^{-1}$ with a corresponding minimum detectable of $7.8 \times 10^{-5} \mathrm{RIU}$.

\subsection{Gas sensing}

In 2001, Stewart et al. [22] demonstrated the use of a CRD system with a micro-optical gas cell inside the fiber loop to trace gas detection of species with weak near-IR absorption lines. For a $\mathrm{CO}_{2}$ gas cell, it was estimated that the detection in the 100$1000 \mathrm{ppm}$ range required ring-down times in the range $250 \mu \mathrm{s}$ to $3 \mathrm{~ms}$. Experimentally, a ring-down time of $2.6 \mu$ s was obtained and a fiber loop loss of $0.48 \mathrm{~dB}$. In 2003, Vogler et al. [23] developed a fiber-optic cavity sensor based on fiber connectors coated with highly reflective dielectric mirrors, thus forming a Fabry-Perot cavity. This sensor was implemented on a CRD scheme and used for hydrogen diffusion into and out of the cavity. The measured ringdown times during $\mathrm{H}_{2}$ diffusion allowed the determination of the diffusion coefficient of hydrogen in silica, resulting in
$3.02 \times 10^{-5} \mathrm{~m}^{2} / \mathrm{s}$ at $30^{\circ} \mathrm{C}$. Only ten years later, gas sensing was subject of research. In 2013, Zhao et al. [24] proposed instead an acetylene gas cell inside the fiber loop for the measurement of gas concentration in the range $[0-5] \%$, showing ringdown times between 13.7 and $12.3 \mu$ s, i.e., decreasing with increasing gas concentration. Recently, Shimizu et al. [25] reported a CRD-based system for $\mathrm{CO}_{2}$ detection. The fiber loop contained a free-space propagation section in order to detect $\mathrm{CO}_{2}$. The light in the freespace section was coupled in and out by collimator lenses coupled with the singlemode fiber loop. In the $\mathrm{CO}_{2}$ concentration range of [0-0.5]\% ringdown times between 1.96 and $1.27 \mathrm{~ms}$, respectively, could be attained and a resolution of $0.05 \%$ was also achieved.

\section{Conclusions}

This brief review on CRD shows that one of the new lines of research is the use of existing equipment on the market for long range measurement. An example was recently demonstrated, namely, the use of the OTDR for the measurement of physical parameters, allowing the multiplexing of some CRD in series. Another line of investigation is signal processing that makes the CRD a quick acquisition system and allows analysis in real time. The use of the add-signal as a new solution presents greater sensitivity when the sensor is subjected to external parameters. Finally it was presented the CRD in various applications from the measurement of deformation to gas monitoring. It is clearly that its application in the future will in medicine, biomedical and chemical areas of research.

\section{Acknowledgments}

This work was financed by FCT - Fundação para a Ciência e Tecnologia (Portuguese Foundation for Science and Technology) and by ERDF (European Regional Development Fund) through: COMPETE Programme (Operational Programme for Competitiveness) within project FCOMP-01-0124-FEDER-037281; ON.2 - O Novo Norte (Northern Portugal Regional Operational Programme). S.S. received a Pos-Doc fellowship (ref. SFRH/BPD/92418/2013) also funded by FCT.

\section{References}

[1] G. Berden, R. Peeters, G. Meijer, Cavity ring-down spectroscopy: experimental schemes and applications, Int. Rev. Phys. Chem. 19 (2000) 565-607.

[2] J.M. Herbelin, J.A. McKay, M.A. Kwok, R.H. Uenten, D.S. Urevig, D.J. Spencer, et al., Sensitive measurement of photon lifetime and true reflectances in an optical cavity by a phase-shift method, Appl. Opt. 19 (1) (1980) 144-147.

[3] D.Z. Anderson, J.C. Frisch, C.S. Masser, Mirror reflectometer based on optical cavity decay time, Appl. Opt. 23 (8) (1984) 1238-1245.

[4] G. Stewart, K. Atherton, B. Culshaw, Cavity-enhanced spectroscopy in fiber cavities, Opt. Lett. 29 (5) (2004) 442-444.

[5] W.P. Tarsa, D.M. Brzozowski, P. Rabinowitz, K.K. Lehmann, Cavity ringdown strain gauge, Opt. Lett. 29 (12) (2004) 1339-1341.

[6] N. Ni, C.C. Chan, X.Y. Dong, J. Sun, P. Shum, Cavity ring-down long period fiber grating strain sensor, Meas. Sci. Technol., Spec. Issue 18 (2007) 3135-3138.

[7] S. Silva, D.J. Passos, M.B. Marques, O. Frazão, Chirped fiber Bragg grating cavity ring-down for strain sensing using an OTDR, Microw. Opt. Technol. Lett. 57 (6 (2015) 1442-1444.

[8] C. Wang, Fiber ringdown temperature sensor, Opt. Eng. Lett. 44 (3) (2005) 030503.

[9] T. von Lerber, M.W. Sigrist, Cavity-ring-down principle for fiber-optic resonators-experimental realization of bending loss and evanescent-field sensing, Appl. Opt. 41 (18) (2002) 3567-3575.

[10] S. Silva, P. Biswas, S. Bandyopadhyay, P.A. Jorge, M.B. Marques, O. Frazão, Fiberoptic cavity ring down using an added-signal for curvature sensing, IEEE Photon. Technol. Lett. 27 (19) (2015) 2079-2082.

[11] C. Wang, S.T. Scherrer, Fiber ringdown pressure sensors, Opt. Lett. 29 (4) (2004) 352-354.

[12] C. Wang, S.T. Scherrer, Fiber loop ringdown for physical sensor development: 
pressure sensor, Appl Opt. 43 (2004) 6458-6464.

[13] H. Qiu, Y. Qiu, Z. Chen, B. Fu, X. Chen, G. Li, Multimode fiber loop ring down spectroscopy for pressure, Microw. Opt. Technol. Lett. 49 (7) (2007) $1698-1700$.

[14] R.S. Brown, I. Kozin, Z. Tong, R.D. Oleschuk, H.-P. Loock, Fiber-loop ring-down spectroscopy, J. Chem. Phys. 117 (2002) 10444-10447.

[15] P.B. Tarsa, A.D. Wist, P. Rabinowitz, K.K. Lehmanna, Singlecell detection by cavity ring-down spectroscopy, Appl. Phys. Lett. 85 (19) (2004) 4523-4525.

[16] S. Pu, X. Gu, Fiber loop ring down spectroscopy with a long period grating cavity, Opt. Lett. 34 (12) (2009) 1774-1776.

[17] N. Ni, C. Chan, L. Xia, P. Shum, Fiber cavity ring-down refractive index sensor, IEEE Photon. Technol. Lett. 20 (16) (2008) 1351-1353.

[18] K.M. Zhou, D. Webb, M. Farries, N. Hayes, L. Zhang, I. Bennion, Biochemical sensor based on a novel all-fibre cavity ring down spectroscopy technique incorporating a tilted fibre Bragg grating, Opt. Lasers Eng. 47 (2009) 1023-1027.

[19] K.M. Zhou, D.J. Webb, C.B. Mou, M. Farries, N. Hayes, I. Bennion, Optical fibe cavity ring down measurement of refractive index with a microchannel drilled by femtosecond laser, IEEE Photon. Technol. Lett. 21 (22) (2009) 1653-1655.

[20] C. Wang, C. Herath, High-sensitivity fiber-loop ringdown refractive index sensors using single-mode fiber, Opt. Lett. 35 (10) (2010) 1629-1631.

[21] W.C. Wong, W. Zhou, C.C. Chan, X. Dong, K.C. Leong, Cavity ringdown refractive index sensor using photonic crystal fiber interferometer, Sens. Actuators B 161 (2012) 108-113.

[22] G. Stewart, K. Atherton, H. Yu, B. Culshaw, An investigation of an optical fibre amplifier loop for intro-cavity and ring-down cavity loss measurements, Meas. Sci. Technol. 12 (2001) 843-849.
[23] D.E. Vogler, M.G. Muller, M.W. Sigrist, Fiber-optic cavity sensing of hydrogen diffusion, Appl. Opt. 42 (27) (2003) 5413-5417.

[24] Y. Zhao, L. Bai, Q. Wang, Gas concentration sensor based on fiber loop ringdown spectroscopy, Opt. Commun. 309 (2013) 328-332.

[25] H. Shimizu, H. Noriyasu, Measurement of carbon dioxide concentration by fiber-loop ring-down spectroscopy for continuous remote measurement, Japn. J. Appl. Phys. 53 (2014) 116601.

[26] C. Wang, Fiber loop ringdown-a time-domain sensing technique for multifunction fiber optic sensor platforms: current status and design perspectives, Sensors 9 (10) (2009) 7595-7621.

[27] M. Fabian, E. Lewis, T. Newe, S. Lochmann, Optical fibre cavity for ring-down experiments with low coupling losses, Measur. Sci. Technol. 21 (9) (2010) 094034.

[28] H. Waechter, J. Litman, A.H. Cheung, J.A. Barnes, H.P. Loock, Chemical sensing using fiber cavity ring-down spectroscopy, Sensors 10 (3) (2010) 1716-1742.

[29] F. Ye, B. Qi, L. Qian, Continuous-wave fiber cavity ring-down measurements using frequency-shifted interferometry, Opt. Lett. 36 (11) (2011) 2080-2082.

[30] T. Hiraoka, T. Ohta, M. Ito, N. Nishizawa, M. Hori, Optical-fiber-type broadband cavity ring-down spectroscopy using wavelength-tunable ultrashort pulsed light, Japn. J. Appl. Phys. 52 (4R) (2013) 040201.

[31] D.J. Passos, S.O. Silva, J.R.A. Fernandes, M.B. Marques, O. Frazão, Fiber cavity ring-down using an optical time-domain reflectometer, Photon. Sens. 4 (4) (2014) 295-299.

[32] D.J. Passos, S.O. Silva, M.B. Marques, O. Frazao, A new cavity ring-down topology for remote sensing, in: Proceedings of Photonics Conference, 2014 Third Mediterranean, pp. 1-3, IEEE, 2014, May. 